\title{
Composición y diversidad de especies arbóreas en transectos de localidades del bosque siempreverde de tierras bajas del Ecuador
}

\section{(Composition and diversity of tree species in transects of location lowland evergreen forest of Ecuador)}

\begin{abstract}
Resumen:
Jorge Caranqui $\mathrm{A}^{1}$

El estudio se realizó en 9 transectos de 1000m2 de bosque siempreverde de tierras bajas, ubicado en dos localidades de la Costa y una en el oriente ecuatoriano. Se planteó contribuir al conocimiento de la diversidad y composición de plantas arbóreas mayores de $10 \mathrm{~cm}$ de diámetro a la altura del pecho (DAP), además de inferir el estado de conservación de los bosques en base en la composición, el número de especies, índices de diversidad y valor de importancia (IV) localizadas en 9 transectos de $1000 \mathrm{~m}^{2}$ de bosque. Se encontraron 156 especies, 107 géneros y 39 familias distribuidas en los 9 transectos, en cada uno la diversidad varía según el índice de diversidad de Simpson de $0.95-0.88$, en este caso todos son diversos porque se aproxima a 1. La mayoría de las especies no están presentes en todos los transectos, el índice de valor en cada transecto no sobrepasa el $40 \%$. Agrupando los transectos coinciden con las tres localidades efectuadas a excepción del transecto 5 y 8 que fueron realizados en sitios perturbados, es decir la mayoría de transectos tienen una perturbación intermedia por eso sus altos índices de diversidad.
\end{abstract}

Palabras clave: Bosque siempreverde, biodiversidad, similitud

\begin{abstract}
:
The study was conducted in 9 transects $1000 \mathrm{~m} 2$ of lowland evergreen forest, located in two locations on the coast and one in eastern Ecuador. It was to contribute to knowledge of the diversity and composition of woody plants over $10 \mathrm{~cm}$ diameter at breast height (DBH) plus infer the state of conservation of forests based on the composition, the number of species, indices diversity and importance value (IV), found in 9 transects of $1000 \mathrm{~m}^{2}$ of forest: 156 species, 107 genera and 39 families distributed in 9 transects, in each one the Simpson diversity index is of 0.92 to 0.95 , in this case are diversity because all approaches 1 . Most were found species aren't present in all transects, the index value in each transect does not exceed $40 \%$. Grouping transects match three locations exception made to transect 5 and 8 were conducted in disturbed sites, the most transects are intermediate disturbance that their high levels of diversity.
\end{abstract}

Keywords: Lowland evergreen forest, biodiversity, similarity

\section{Introducción}

El bosque siempreverde de tierras bajas clasificado por Harling (1986) como bosque lluvioso de las tierras bajas, cubre el norte de las tierras bajas de la costa del Pacífico bajo los $700 \mathrm{~m}$ de elevación, la mayor parte de Esmeraldas, las áreas adyacentes de la provincia de Pichincha y áreas pequeñas del norte de Manabí y Los Ríos. Este tipo de vegetación también cubre

\footnotetext{
${ }^{1}$ Escuela Superior Politécnica del Chimborazo, Riobamba - Ecuador (jorge.caranqui@espoch.edu.ec)
} 
virtualmente todas las tierras bajas de la Amazonía al este de los Andes. También se caracteriza por un clima con una precipitación anual por sobre los 3000 mm., y carece de una estación seca marcada (es decir, generalmente no más de un mes con menos de $100 \mathrm{~mm}$ de precipitación). Este es el tipo de vegetación más extenso en el país, que cubre más de un tercio del Ecuador continental (Neill 1999).

El bosque siempreverde de tierras bajas es alto, denso y siempre verde, con el dosel frecuentemente de $30 \mathrm{~m}$., o más de altitud y una diversidad alta de especies. La diversidad alfa de los árboles, como se muestra en las parcelas permanentes de una hectárea, es más alta en la Amazonía ecuatoriana que en el área del bosque lluvioso en el norte de la costa del Pacífico (Neill, 1999). El bosque siempreverde de tierras bajas del noroeste del Ecuador es muy similar al de la región del Chocó colombiano de la costa del Pacífico y comparte muchas especies, pero hay también un elemento significativo de especies endémicas que no se conocen al norte de la frontera colombiana (Neill, 1999); muchas especies están también distribuidas en los bosques húmedos de la Amazonía y/o en América Central (Valencia et al., 1998).

Por lo manifestado anteriormente, el objetivo de este trabajo es contribuir al conocimiento de la composición y diversidad de especies leñosas mayor de $10 \mathrm{~cm}$ de diámetro a $1.30 \mathrm{~m}$ sobre el suelo (DAP), encontrada en 9 transectos (unidades muestreales) del "bosque lluvioso de tierras bajas, en dos localidades de la Costa y una en la Amazonía basados en el "IV" (índice de valor), diversidad y similitud.

\section{Materiales y métodos}

\section{1. Área de estudio}

El presente estudio se realizó en 3 localidades (tabla 1) de la Costa y de la Amazonía ecuatoriana que pertenece a la formación de "bosque siempreverde de tierras bajas" (Sierra, 1999).

Tabla 1. Ubicación geográfica de los transectos en estudio.

\begin{tabular}{|l|l|l|l|l|l|}
\hline TRANSECTO & ALTITUD msnm) & COORDENADAS & PROVINCIA & CANTÓN & LOCALIDAD \\
\hline T1-T5 & 200 & $00^{\circ} 35^{\prime} \mathrm{S}, 79^{\circ} 21^{\prime} \mathrm{W}$ & Los Ríos & Buena Fé & Río Palenque \\
\hline T6-T8 & 240 & $00^{\circ} 08^{\prime} \mathrm{S}, 76^{\circ} 45^{\prime} \mathrm{W}$ & Sucumbios & Shushufindi & Incinerox \\
\hline T9 & 400 & $02^{\circ} 17^{\prime} \mathrm{S}, 79^{\circ} 05^{\prime} \mathrm{W}$ & Chimborazo & Cumandá & Chilicay \\
\hline
\end{tabular}

\subsection{Toma de datos}

Durante el 2012 y 2013, se procedió a inventariar 9 transectos de $1000 \mathrm{~m}^{2}$, en forma de zig-zag cada uno con cinco líneas de 50x4 m cada una con rumbo al Norte (Caranqui, 2011); (Cerón ,2003); (Phillips, 2002), ubicados dentro de las zonas de estudio, éstas zonas se escogieron por 
las facilidades brindadas por sus propietarios. Se midieron todos los árboles iguales o mayores a $10 \mathrm{~cm}$ de DAP. Se colectaron especímenes de los individuos fértiles. Las muestras se prensaron y fueron secadas e identificadas por el autor en el Herbario de la Escuela Superior Politécnica de Chimborazo (CHEP), y verificado la información en Jorgensen (1999) y en la base de datos (Missouri Botanical Garden).

\subsection{Análisis de datos}

Se realizaron los siguientes cálculos (Cerón, 2003):

Área Basal (AB)

$$
\mathrm{AB}=\frac{\pi(\mathrm{D})^{2}}{4}
$$

Donde

$\mathrm{AB}=$ Área basal

$\mathrm{D}=\mathrm{DAP}$ [Diámetro a la altura del pecho $(1.30 \mathrm{~m})$.

$\pi=3.1416($ constante)

Densidad = Número de árboles en la parcela

Densidad Relativa (DR)

$$
\mathrm{DR}=\frac{\# \text { de árboles de una especie }}{\# \text { árboles en la parcela }} \times 100
$$

Dominancia Relativa (DMR)

$$
D M R=\frac{\text { área basal de una especie }}{\text { área basal total de la parcela }} \times 100
$$

Índice de Valor (IV)

$$
I V=D R+D M R
$$

Además en cada uno de los transectos con sus respectivas frecuencias se obtuvieron: riqueza, diversidad (índice de Simpson), e índice de similitud (Bray Curtis, 1957), calculados en el programa PAST. 
Según (Golicher, 2012), la fórmula para el índice de Simpson es:

$$
D=\frac{\sum_{i=1}^{S} n_{i}\left(n_{i}-1\right)}{N(N-1)}
$$

Donde $S$ es el número de especies, $N$ es el total de organismos presentes (o unidades cuadradas) y $n$ es el número de ejemplares por especie.

En la ecología y la biología , la disimilitud Bray-Curtis (1957), es una estadística que se usa para cuantificar la disimilitud en la composición entre dos sitios diferentes, basadas en conteos en cada sitio. Según la definición de Bray y Curtis, el índice de similitud:

$$
B C_{i j}=\frac{2 C_{i j}}{S_{i}+S_{j}}
$$

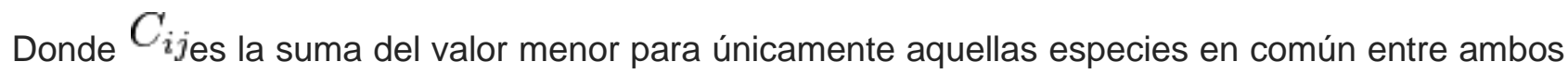

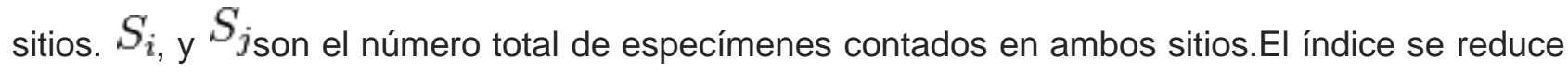
a $2 \mathrm{C} / 2$ = C, donde la abundancia en cada sitio se expresan como un porcentaje. El tratamiento adicional se puede encontrar en Legendre y Legendre. La disimilitud de Bray-Curtis está directamente relacionada con el índice de similitud de Sørensen $Q S_{i j}$ entre los mismos sitios:

$$
\overline{B C}_{i j}=1-Q S_{i j}
$$

La disimilitud de Bray-Curtis está obligado entre 0 y 1, donde 1 significa que los dos sitios tienen la misma composición (es decir que comparten todas las especies), y 0 significa que los dos sitios no comparten ninguna especie. En los sitios con donde $A C$ es intermedia (por ejemplo, $A C=0,5$ ) este índice se diferencia de otros índices comúnmente utilizados.

\section{Resultados}

\subsection{Composición florística}

En total se obtuvo 156 especies,107 Géneros y 39 familias, las familias con más especies son: Moraceae con 18, Fabaceae con 16 especies, Urticaceae con 12, Meliaceae con 10, Rubiaceae con 8, Euphorbiaceae con 7, Arecaceae, Myristicaceae, Sapotaceae y Melastomataceae con 6; el resto de familias con valores menores. Estas diez familias mencionadas (de las 39 familias en total), contienen 95 especies es decir el $60.89 \%$ del total de familias.

En la Tabla 2 se encuentra las treinta especies con mayores índices de valor (IV), distribuidas en los 9 transectos, en la mayoría de los casos no se repiten las especies en los transectos, las especies que lo hacen con mayor frecuencia son: Ficus tonduzii Standl., Guarea kunthiana A.Juss, en 5 de los 9 , Trichilia pleeana (A.Juss.) C.DC., en dos de los 9 transectos pero con 
valores mayor de $25 \%$ "IV", El resto de especies no tienen representabidad en menos del $20 \%$ de los transectos en estudio.

Tabla 2. Listado de especies con sus respectivos índices de valor de importancia (IV) en los 9 transectos:

T1-T5: Río Palenque; T6-T8: Shushufindi; T9: Chilicay.

\begin{tabular}{|c|c|c|c|c|c|c|c|c|c|c|}
\hline Familias & Especies & T1 & T2 & T3 & T4 & T5 & T6 & T7 & T8 & T9 \\
\hline Meliaceae & Trichilia pleeana (A.Juss.) C.DC. & 0 & 0 & 0 & 0 & 0 & 30,3 & 24,8 & 0 & 0 \\
\hline Moraceae & Ficus tonduzii Standl. & 15,4 & 8,21 & 3,5 & 4,57 & 0 & 4,16 & 16,5 & 0 & 0 \\
\hline Urticaceae & Cecropia sp. 2 & 0 & 0 & 0 & 0 & 39,6 & 0 & 0 & 0 & 0 \\
\hline Moraceae & Castilla elástica Sessé & 0 & 0 & 12,4 & 22,4 & 0 & 0 & 0 & 0 & 0 \\
\hline Cannabaceae & Trema micranta (I.) Blume & 0 & 0 & 27,4 & 0 & 0 & 0 & 0 & 0 & 0 \\
\hline Fabaceae & Erytrina edulis Triana ex Micheli & 0 & 10,7 & 0 & 0 & 11,3 & 0 & 0 & 0 & 0 \\
\hline Sapotaceae & Pouteria sp. & 3,45 & 16,9 & 0 & 0 & 0 & 0 & 0 & 0 & 0 \\
\hline Urticaceae & Cecropia sciadophylla Mart. & 0 & 0 & 0 & 18,2 & 0 & 0 & 0 & 0 & 0 \\
\hline Malvaceae & Ceiba pentandra (L.) Gaertn. & 0 & 0 & 0 & 0 & 0 & 0 & 0 & 17,7 & 0 \\
\hline Fabaceae & Inga edulis Mart. & 0 & 0 & 0 & 0 & 0 & 0 & 0 & 17 & 0 \\
\hline Meliaceae & Ruagea pubescens (Rich.) A.Juss. & 0 & 0 & 0 & 0 & 0 & 4,38 & 1,73 & 0 & 10 \\
\hline Moraceae & Batocarpus orinoscensis H.Karst. & 0 & 0 & 0 & 0 & 0 & 7,38 & 5,44 & 0 & 2,7 \\
\hline Urticaceae & Cecropia sp. & 10,8 & 4,65 & 0 & 0 & 0 & 0 & 0 & 0 & 0 \\
\hline Salicaceae & $\begin{array}{l}\text { Tetrathylacium macrophyllum } \\
\text { Poepp. }\end{array}$ & 0 & 0 & 0 & 0 & 0 & 0 & 0 & 0 & 14,1 \\
\hline Myristicaceae & $\begin{array}{l}\text { Otoba gracilipes (A.C.Sm.) } \\
\text { A.H.Gentry }\end{array}$ & 0 & 0 & 13,4 & 0 & 0 & 0 & 0 & 0 & 0 \\
\hline Araliaceae & $\begin{array}{l}\text { Dendropanax arboreus (L.) Decne. } \\
\text { \& Planch. }\end{array}$ & 0 & 0 & 0 & 0 & 0 & 10,1 & 2,06 & 0 & 0 \\
\hline Moraceae & Clarisia racemosa Ruíz \& Pav. & 0 & 1,65 & 0 & 4,56 & 3,38 & 0 & 2,5 & 0 & 0 \\
\hline Fabaceae & Myroxylon balsamum (L.) Harms & 0 & 0 & 0 & 0 & 0 & 3,7 & 4,18 & 3,96 & 0 \\
\hline Meliaceae & Carapa guianensis Aubl. & 0 & 1,46 & 0 & 0 & 0 & 0 & 0,53 & 0 & 9,67 \\
\hline Moraceae & Sorocea puvibena & 0 & 0 & 0 & 0 & 0 & 0 & 0 & 0 & 11,6 \\
\hline Myristicaceae & Virola elongata (Benth.) Warb. & 0 & 0 & 0 & 0 & 0 & 10,5 & 0 & 0 & 0,8 \\
\hline Urticaceae & Cecropia obtusifolia Bertol. & 1,89 & 0 & 9,27 & 0 & 0 & 0 & 0 & 0 & 0 \\
\hline Moraceae & Maclura tinctoria (L.) Steud. & 0 & 1,55 & 4,01 & 5,38 & 0 & 0 & 0 & 0 & 0 \\
\hline Fabaceae & Inga sp.3 & 1,49 & 6,15 & 1,91 & 1,18 & 0 & 0 & 0 & 0 & 0 \\
\hline Indeterminada & Indeterminada3 & 0 & 0 & 0 & 10,2 & 0 & 0 & 0 & 0 & 0 \\
\hline Boraginaceae & $\begin{array}{l}\text { Cordia alliodora (Ruiz \& Pav.) } \\
\text { Oken }\end{array}$ & 0 & 0 & 0 & 0 & 0 & 0 & 0 & 9,45 & 0 \\
\hline Meliaceae & Trichilia cipo (A.Juss.) C.DC. & 0 & 0 & 0 & 0 & 0 & 0 & 9,4 & 0 & 0 \\
\hline Moraceae & Ficus macbridei Standl & 0 & 0 & 9,4 & 0 & 0 & 0 & 0 & 0 & 0 \\
\hline Meliaceae & Guarea kunthiana A.Juss. & 1,43 & 0 & 0 & 0 & 0 & 0,95 & 0,81 & 1,14 & 4,96 \\
\hline Meliaceae & Guarea ptorrahchis Harms & 0 & 0 & 0 & 0 & 0 & 0 & 9,23 & 0 & 0 \\
\hline
\end{tabular}

Con respecto al mayor valor de "IV", pertenece a la especie Cecropia sp.2 con 39,56\% que solo está en el T5, Trichilia pleeana (A.Juss.) C.DC. con 30.32\% en el T6 y 24.84 en el T7, Ficus tonduzii Standl con el 16,47 en el T7. Como se puede apreciar entre los 10 "IV" altos solo Cecropia sp.2 tiene valor alto en un solo transecto; si a los 10 "IV" mayores agrupamos en las 
familias respectivas, tendremos que las familias más representativas son Urticaceae, Moraceae y Fabaceae con 2 especies y el resto se distribuyen en familias como Cannabaceae, Sapotaceae, Malvaceae y Meliaceae.

\subsection{Diversidad}

El listado de especies (Tabla 2) se ingresó en el programa PAST generándose el índice de diversidad de Simpson que fluctúa de 0.92- 0.95 (Tabla 3), el cual resulta que los valores son altos en base al número de especies e individuos en cada transecto ya que todos los valores son cercanos a 1. En cuanto a la relación entre el número de especies y de individuos no encontramos un patrón común, ya que los transectos con alto número de especies e individuos no alcanzan los altos valores del índice de diversidad.

Tabla 3. Datos de los cinco transectos con datos de abundancia y diversidad: T1-T5: Río Palenque; T6-T8: Shushufindi; T9: Chilicay

\begin{tabular}{|l|l|l|l|l|l|l|l|l|l|}
\hline & T1 & T2 & T3 & T4 & T5 & T6 & T7 & T8 & T9 \\
\hline Especies & 26 & 27 & 6 & 22 & 21 & 24 & 29 & 23 & 23 \\
\hline Individuos & 42 & 45 & 35 & 51 & 64 & 80 & 101 & 47 & 70 \\
\hline I. Simpson & 0,95 & 0,95 & 0,92 & 0,92 & 0,93 & 0,92 & 0,93 & 0,94 & 0,93 \\
\hline
\end{tabular}

\subsection{Similitud}

La Figura 1 indica que hay 4 grandes grupos:1) integrado por los transectos 1-4, 2) conformado por el T5, 3), conformado por T8; tanto el T5 y el T8 fueron tomados de sitios disturbados y 4), conformado por T6-T7 y T9.

Hay varios subgrupos que tienen dicotomía o cierta dicotomía, generalmente se agrupan por localidad es decir 1-4: Río Palenque, 6-7: Shushufindi, y el T9 no tiene dicotomía por ser un transecto en localidad única; como mencionamos el T5 y T8 son muy diferentes del resto por ser de sitios disturbados.

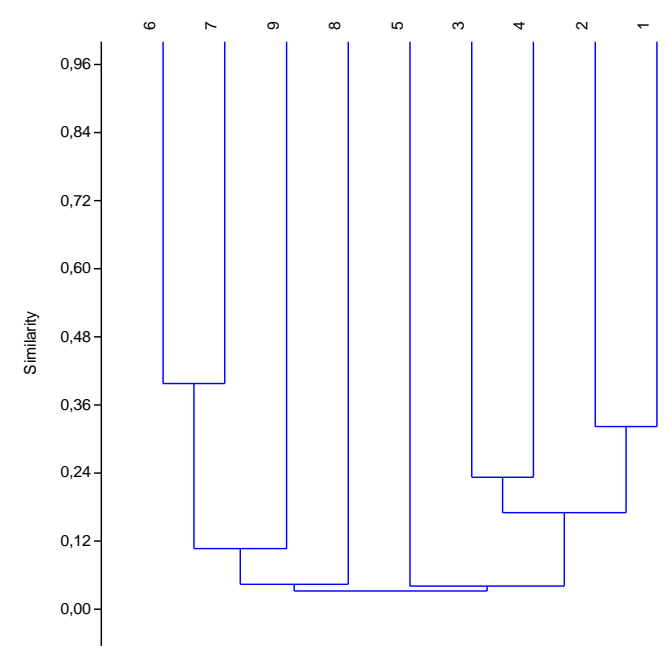

Figura 1. Dendrograma del índice de Bray- Curtis con los 14 transectos en estudio 


\section{Discusión}

Según Jorgensen \& León Yánez (1999), la comparación de la composición de especies en las cuatro diferentes regiones revela bajos niveles de similitud entre ellas pero la similitud más alta se encuentra entre la Amazonía y la Costa. Por tal razón en términos generales, a nivel de familias coiciden con los grandes grupos encontrados como Fabaceae, Moraceae, Melastomataceae, Meliaceae, Rubiaceae, Urticaceae (Cecropia), Euphorbiaceae y Arecaceae coincidiendo con otros estudios: Cerón (1997), Balslev (1987), Romero et-al (2001), Phillips (2003), Bonifaz \& Cornejo (2004).

Los valores del "IV" no superan el $40 \%$, esto puede suponer que las especies se encuentran distribuidas por todo el bosque pero, como ocurre en toda comunidad diversa, su abundancia es poco equitativa (Hubbell \& Foster, 1992).

El índice de Simpson indica la relación entre riqueza o número de especies y la abundancia o número de individuos por especies en cualquier sitio dado (Moreno, 2001; Smith \& Smith, 2003); excepto para el caso de unas pocas especies de una comunidad no hay ninguna relación entre la riqueza de una comunidad (número de individuos) y su diversidad En los transectos en estudio obtuvimos índices que van de 0.92 a 0.95 que es una diversidad alta como en Conditt (2002), Romero (2001), Pitman (2001) y Valencia (2004). La "hipótesis de la perturbacion intermedia" en Rabinowrtz (1986), propone que en términos de la composición de especies una comunidad nunca alcanza el equilibrio y que una alta diversidad es el resultado de un constante cambio en las condiciones del ecosistema. El modelo de la perturbación intermedia postula que la diversidad de especies alcanza su máximo cuando las perturbaciones ocurren a frecuencias e intensidades "intermedias"; es decir a perturbaciones intermedias mayor diversidad, es por ello que en las zonas de estudio se pudo observar bosque secundarios con distinto grado de conservación que se han agrupado en menor o mayor grado excepto el T5 y T8 que son bosques disturbados lo corrobora el dendrograma de Bray Curtis (Fig.1).

A comparación de otros ecosistemas en el Ecuador, los bosques húmedos tropicales amazónicos tienen muchos estudios, pero la mayoría de ellos es en base a parcelas permanentes de $1 \mathrm{Ha}$ (Ter teege et-al 2013; Conditt et-al 2002; Valencia et-al 2004). Cuando se popularizaron los estudios de diversidad de bosques en el Ecuador la metodología empleada fue diseñada por Alwin Gentry (Phillips 2003), que despertó el interés para hacer estudios de mayor tamaño; no por esto deberían ser desestimados actualmente los estudios de transectos (Caranqui, 2011), ya que $1000 \mathrm{~m}^{2}$ es un área mínima para encontrar información importante sobre ecología de los bosques amazónicos (Romero, 2001; Phillips, 2003), es ideal hacer parcelas de 1Ha, pero ello conlleva a mayor recursos. En este estudio se ha confirmado que los datos cuantitativos (familias), y conceptos ecológicos coiciden con los datos en las que se ha realizado en una hectárea. 


\section{Conclusiones y recomendaciones}

La biodiversidad de los transectos según el índice de diversidad de Simpson es alto entre los transectos estudiados (0.87-0.95). Pero al comparar los transectos con las especies y sus valores los transecto 5 y 8 son diferentes al resto de transectos; esto se debe a la perturbación intermedia que sufren estos bosques.

Con los resultados presentados la familia Fabaceae tienen los mayores valores y coinciden con los estudios existentes de este tipo de bosque.

\section{Agradecimientos}

Doy fe de agradecimiento a las siguiente personas que colaboraron con la realización de este documento: Blgo. Fredy Villao, Dr. Bryan Maclaren, Blgo Xavier Cornejo, Dr. Tim McDowel, Ing. Marcelo Pino, Msc. Alina Freire, estudiantes de Maestría Forestal UTEQ 2012.

\section{Bibliografía}

Balslev, H., Luteyn, J., Øllgaard, B., \& Holm-Nielsen, L. B. (1987). Composition and structure of adjacent unflooded and floodplain forest in Amazonian. Ecuador.Opera Botanica, 92, 37-57

Bonifaz, C., y X. Cornejo. (2004). Flora del bosque de Garúa en Loma Alta. Universidad de Guayaquil. Páginas: 8-17

Bray, J. R \& , J. T.Curtis. (1957). An ordination of the upland forest communities of southern. Wisconsin. Ecological monographs, 27(4): 325-349

Caranqui, J. (2011). Estudios básicos de bosques montanos en el centro del Ecuador. Editorial Académica Española. 67 páginas. Publicado en Alemania.

Cerón, C.E., (2003). Manual de Botánica ecuatoriana: Sistemática y Métodos de estudio". Facultad de Filosofía. Letras y Educación. Universidad Central del Ecuador. Quito - Ecuador. Pp.281282.

Cerón, C. E., \& Montalvo, C. (1997). Composición y estructura de una hectárea de bosque en la Amazonía Ecuatoriana, con información etnobotánica de los Huaorani. Estudios sobre diversidad y ecología de plantas. Pontificia Universidad Católica del Ecuador publicaciones. Quito, 153-172.

Condit, R., Pitman, N., Leigh, E. G., Chave, J., Terborgh, J., Foster, R. B., ... \& Hubbell, S. P. (2002). Beta-diversity in tropical forest trees. Science, 295(5555), Pp.666-669. 
Denslow, J.S.. (1985). Disturbance mediated coexistence os species. En The ecology of natural disturbance and patch dynamics(307-323). USA: Academic press.

Golicher, D . (2012). ¿Cómo cuantificar la diversidad de especies?. Tomado de: http://www.dfpd.edu.uy/cerp/cerp_norte/cn/Biologia/BIODIV/Como\%20cuantificar\%20la\%20d iversidad,\%20algunos\%20ejercicios.pdf

Hubell, S.P. and R.B. Foster.( 1992). Short-term dynamics of a neotropical forest: whith ecological research matters to tropical conservation and management. Oikos 63: 48- 54.

Jørgensen, P. M . and S. León-Yánez. (1999). "Catalogue of the vascular plants of Ecuador". Missouri Botanical Garden.

MacArthur, R. H., \& E. O. Wilson. (1963). An equilibrium theory of insular zoogeography. Evolution, 17(4), 373-387.

Missouri Botanical Garden. (2013, Nov.) Base de datos trópicos. (En línea). Disponible en htpp://www.tropicos.org.

Moreno, C.E. (2001). Métodos para medir la Biodiversidad. M \& T- Manuales y Tesis SEA, vol. ¡. Zaragoza- España.. 84 pag.

Neill, D. Vegetación. (1999). En Catálogo de plantas vasculares del Ecuador, J.Jorgensen \& S.León-Yañez (ed.). (2002). Saint Louis Missouri. P. 13-17

Phillips, O., and J. S. Miller. (2002). Global patterns of plant diversity: Alwyn H. Gentry's forest transect data set. Monographs in Systematic Botany from the Missouri Botanical Garden 89: 1-319.

Pitman, N. C., Terborgh, J. W., Silman, M. R., Núñez V, P., Neill, D. A., Cerón, C. E., \& Aulestia, M. (2001). Dominance and distribution of tree species in upper Amazonian terra firme forests. Ecology, 82(8),Рp.: 2101-2117.

Rabinowrtz, D., Cairns, S., \& Dillon, T. (1986). Seven kinds of rarity,pags. 182-204. In M.E.Soule(Editor). ConservationBiology.Sinauer,Sunderland,Massachuses.RZ.

Romero-Saltos, H., Valencia, R., \& Macía, M. J.( 2001). Patrones de diversidad, distribución y rareza de plantas leñosas en el Parque Nacional Yasuní y la Reserva Étnica Huaorani, Amazonía ecuatoriana. Evaluación de productos forestales no maderables en la Amazonıa noroccidental, 131-162. 
Sierra, R. (1999). "Propuesta Preliminar de un Sistema de Clasificación de Vegetación para el Ecuador Continental". Quito - Ecuador. Pp.63-64

Smith, L and T. Smith. (2001). Ecología. Pearson Education. Madrid.. Pag. 664.ter Steege, H., Pitman, N. C., Sabatier, D., Baraloto, C., Salomão, R. P., Guevara, J. E.,\& Fine, P. V. (2013). Hyperdominance in the Amazonian tree flora. Science, 342(6156).

Valencia, R., H., Balslev, W., Palacios, D., Neill., C. Josse., M. Tirado \& F. Skov. (1998).

Diversity and family composition of tress in different regions of Ecuador: a sample of 18 onehectare plots. Pp 569-584. En F. Dallmeier and J. A. Comiskey(eds.). Forest Biodiversity in North,Central and South America and the Carribean: Research and Monitoring.Man and the Biosphere Series 21. Unesco and The Parthenon Publishing Group.Carnforth.

Valencia, R., Foster, R. B., Villa, G., Condit, R., Svenning, J. C., Hernández, C., \& Balslev, H. (2004). Tree species distributions and local habitat variation in the Amazon: large forest plot in eastern Ecuador. Journal of Ecology, 92(2),Pp.: 214-229. 